\title{
Recombinant human endostatin reduces hypertrophic scar formation in rabbit ear model through down- regulation of VEGF and TIMP-1.
}

\author{
Peng Wang ${ }^{1}$, Li-Zhu Jiang ${ }^{2}$, Bin Xue ${ }^{3}$
}

1. Department of Orthopedics, First Affiliated Hospital of Chongqing Medical University, Chongqing, China; wangpeng.2015@hotmail.com

2. Department of Otolaryngology, First Affiliated Hospital of Chongqing Medical University, Chongqing, China; pear11984@sina.com

3. Department of Burn and Plastic Surgery, First Affiliated Hospital of Chongqing Medical University, Chongqing, China; CQXuebin@126.com

\begin{abstract}
Background: Recombinant human endostatin (Endostar) has been widely used to suppress angiogenesis in carcinoma patients. Hypertrophic scar (HS) tissue, much like a carcinoma, is often associated with angiogenesis. However, there have been few studies conducted on the effects of Endostar on HS or its mechanism.

Objective: This paper investigated the effects Endostar on the HS of rabbit ears and studied the effects of Endostar on VEGF and TIMP-1 expression.

Methods: Sixteen New Zealand white rabbits were used to establish HS models. Then, rabbit ears containing HS were randomly assigned to either the Endostar group or the control group. The changes of appearance and histology were evaluated using the naked eye, hematoxylin eosin staining, and a scar elevation index. The VEGF and TIMP-1 expressions were detected by immunohistochemical staining, RT-PCR, and western blot.

Results: The thickness of the connective tissue in the Endostar group were thinner, the numbers of micro vessels and fibroblasts were fewer, and the collagen fibers were smoother. Moreover, the mRNA and protein expressions of VEGF and TIMP-1 in the Endostar group were significantly lower than those in the control group.

Conclusion: The results suggested that Endostar reduced the formation of HS by down-regulation of VEGF and TIMP-1 expressions.

Keywords: Endostar, endostatin, hypertrophic scar, vascular endothelial growth factor, tissue inhibitor of metalloproteinase-1 DOI: http://dx.doi.org/10.4314/ahs.v16i2.23

Cite as: Wang P, Jiang L-Z, Xue B. Recombinant human endostatin reduces hypertrophic scar formation in rabbit ear model through down- regulation of VEGF and TIMP-1. Afri Health Sci 2016;16(2): 542-553. http:// dx.doi.org/10.4314/ahs.v16i2.23
\end{abstract}

\section{Introduction}

A hypertrophic scar (HS), an excessive healing reaction to injury, is characterized by excessive fibrosis and collagen deposition in extracellular matrix $(\mathrm{ECM})^{1}$. Surgical excision, intralesional injection of steroids, radio frequency, radiation, and use of lasers are the most frequently used

\section{Corresponding author:}

Bin Xue,

Department of Burn and Plastic Surgery,

First Affiliated Hospital of Chongqing Medical

University, Chongqing, China

Email: CQXuebin@126.com treatments for $\mathrm{HS}^{2}$. Nevertheless, there is no consensus on which treatment is the most effective with the fewest side effects. Therefore, novel strategies for the prevention and treatment of HS are worthy of further investigation toward the goal of its occurrence being avoided entirely.

Recent research shows that HS is associated with the abnormal proliferation of fibroblasts and the overproduction of collagen and extracellular matrix ${ }^{1,3}$. In addition, angiogenesis plays an essential role in the early stages of wound healing, and micro vascular abnormality is found in pathological scars ${ }^{3}$. Moreover, research suggests that HS has increased vascular components compared to normal dermis or scars ${ }^{4-6}$. Thus, it was theorized that anti- 
angiogenesis is the key to inhibiting the formation of HS. Endostar is a modified human endostatin with a nine amino acid sequence at the $\mathrm{N}$-terminus (MGGSHHHHH). It is widely used in suppressing angiogenesis in carcinomaand is far more efficient than previous endostatins ${ }^{7}$. Although HS presents angiogenesis much like carcinoma, there are limited studies regarding the effects of Endostar on HS and its molecular mechanism.

Vascular endothelial growth factor (VEGF), a positive regulator of angiogenesis, is produced in large amounts by epidermal cells during wound healing. ${ }^{8}$. The over expression of VEGF has been demonstrated in early stages of pathological scar formation ${ }^{9,10}$. Inhibition of VEGF activity with the injection of anti-VEGF antibodies has been shown to decrease capillary formation, collagen I production, and overall scar volume ${ }^{11}$. Although the over expression of VEGF has shown to be significantly correlated with the formation of scars, the relationship between Endostar and VEGF remains undetermined in HS.

The major effectors of ECM degradation and remodeling belong to a family of structurally related enzymes called matrix metalloproteinases (MMPs). Endogenous inhibitors of MMPs are tissue inhibitors of metalloproteinases (TIMPs). Increased levels of TIMP-1 have been found in patients with HS, suggesting that elevated systemic TIMP-1 concentrations might contribute to tissue fibrosis, leading to HS formation ${ }^{12}$.
Excessive expressions of TIMP-1 and VEGF have been shown to be correlated with pathological scar formation. Because HS is associated with the occurrence of angiogenesis and Endostar is an angiogenesis inhibitor, this study aimed to investigate the effects of Endostar on HS tissue in model of rabbit ears and the effects of Endostar on VEGF and TIMP-1 expressions.

\section{Materials and methods \\ Ethics statement}

All animals were studied according to the guidelines of the Declaration of Helsinki, and experiments were carried out with the approval of the Animal Experimentation Ethics Committee of Chongqing Medical University.

\section{Establishment of model rabbit ears}

Sixteen male adult New Zealand white rabbits weighing between 2.2 and $2.5 \mathrm{~kg}$ were selected for this study. The rabbit HS models were established according to the previous report ${ }^{13}$. The animals were anaesthetized with $1 \%$ $(10 \mathrm{~g} / \mathrm{L})$ pentobarbital sodium $(1 \mathrm{mg} / \mathrm{kg})$, and then one hundred ninety-two wounds were created on the ventral surface of each ear by means of an $11 \mathrm{~mm}$ pen cover (Figure 1). An operating loupe was used to ensure the removal of the epidermis, dermis, and perichondrium in each wound. Thereafter, the wounds were cleaned every day to remove the crust and stimulate the growth of granulation. After 25 days, 128 rabbit HSs had formed. Two wounds on the tail of each ear were discarded because no HS had formed according to the scar elevation index.

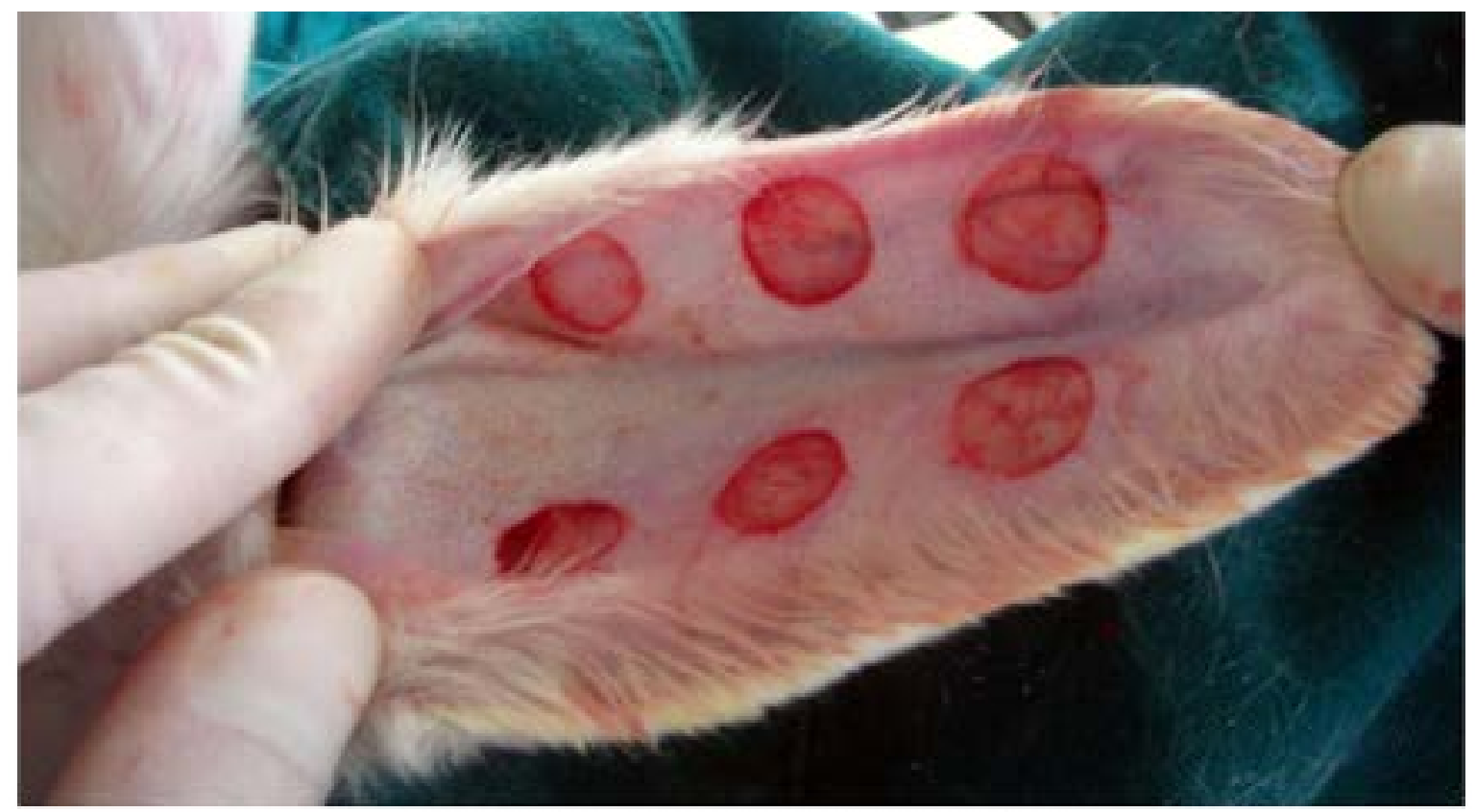

Figure 1. Formation of punch defects 


\section{Treatment}

Thirty-two ears, which contained a total of $128 \mathrm{HSs}$, were randomly assigned to the Endostar group and the control group. The control group was treated with saline injections, and the Endostar group was treated with Endostar injections ( $5 \mathrm{mg} / \mathrm{mL}$, Simcere Pharmaceutical Group, China). Each scar was injected with $1 \mathrm{~mL}$ solution once every two days for a total of 5 treatments. After 30 days, the rabbits were euthanized and the scars were harvested.

\section{Scar elevation index}

Scar elevation index (SEI) is a ratio of the total height of the wound area tissue to the area of normal tissue below the scar. The height of the normal tissue is determined based on the height of the adjacent unwounded dermis. A SEI of 1 indicates that no newly hypertrophied dermis has formed, whereas an index $>1$ indicates the formation of $\mathrm{HS}^{14}$.

\section{Hematoxylin - Eosin staining}

The samples were fixed with $10 \%$ buffered formalin, dehydrated, embedded in paraffin, cut in $5 \mathrm{~mm}$ sections, and stained with haematoxylin and eosin (H\&E).

\section{Quantitative real-time RT-PCR}

Twenty randomly selected tissues from the Endostar group and twenty from the control group were selected for immunohistochemistry examination and the VEGF and TIMP expressions at the RNA level were evaluated. Total RNA was extracted using Trizol reagent (Takara, Dalian, China) according to the manufacturer's instructions, and contaminating genomic DNA was removed by incubation with DNase I (Takara, Dalian, China). RNA purity and concentration were determined by spectrophotometry. PCR was carried out according to the standard protocol on a real-time PCR-system (Applied BioSystems) with SYBR Green detection. After an initial incubation of the $10 \mu \mathrm{l}$ reaction mixture for $1 \mathrm{~min}$ at $95^{\circ} \mathrm{C}, 39$ cycles $\left(95^{\circ} \mathrm{C}\right.$ for $20 \mathrm{~s}, 58^{\circ} \mathrm{C}$ for $\left.25 \mathrm{~s}\right)$ were performed for amplification. The specificity of amplification was confirmed by melting curve analysis. Each sample was tested in triplicate and the results were normalized to the level of gene GAPDH. The sequences of each set of primers were as follow: 5'-CATATTCAAGCCT'TCCTGC-3' (sense) and 5'-GGTCTGCAT'TCACATT'TGTTG-3' (antisense) for VEGF;5'-TGT'TGT'TGCTGTGGCTGATAG-3' (sense) and 5'-CGCTGGTATAAGGTGGTCTGG-3' (anti- sense) for TIMP-1; 5'-CAGCGACACCCACTCCTC-3' (sense) and 5'-TGAGGTCCACCACCCTGT -3' (antisense) for GAPDH. Relative gene expression was calculated as $2-\Delta \Delta C T$.

\section{Immunohistochemical staining}

Sixty-four tissues from the Endostar group and sixty-four from the control group were collected for immunohistochemical staining, which was performed on $5 \mu \mathrm{m}$ paraffin tissue sections mounted on polylysine-coated slides and dried at $37^{\circ} \mathrm{C}$ overnight. After the slides were deparaffinized in xylene and rehydrated conventionally, the endogenous peroxidase was blocked with 3\% hydrogen peroxide in methanol for 20 minutes. Each slide was incubated with normal goat serum for 20 minutes at room temperature. The sections were incubated with rabbit anti-VEGF polyclonal antibody (1:100 dilution, Boster Biological Technology, Ltd, Wuhan, China) and rabbit anti-TIMP-1 polyclonal antibody (1:100 dilution, Boster Biological Technology, Ltd, Wuhan, China) overnight at $4^{\circ} \mathrm{C}$. After washing with phosphate-buffered saline, sections were incubated for 30 minutes with a horseradish peroxidase labeled polymer anti-rabbit secondary antibody (Boster Biological Technology). Chromogen 3, 3-diaminobenzidine (Boster Biological Technology) was used for 15 minutes to visualize immunolabeling, resulting in a brown precipitate. After washing, the sections were counterstained with hematoxylin. Positive and negative immunohistochemistry controls were routinely performed.

Staining intensity was given one of the following four grades: none (0), weak (1), moderate (2), or strong (3). The percentage of positive cells was also given one of the following four grades: 0 (<1\%), 1 (2\%-20\%), 2 (21\%-50\%), $3(51 \%-100 \%)$. The staining result was semi-quantitatively assessed by the score combined with staining intensity and the percentage of positive cells ${ }^{15}$. An express severity score less than 2 was negative (-), 2-3 was weakly positive $(+)$, more than 3 was strongly positive $(++)$; the results were then judged according to the score. The scores from two independent investigators were compared and disagreements were resolved by consensus.

\section{Western blot}

Twenty randomly selected tissues from the Endostar group and twenty from the control group were selected 
for immunohistochemistry, and the VEGF and TIMPexpressions at the protein level were evaluated. Protein samples $(40 \mu \mathrm{g})$ were separated on precast $10 \%$ SDS polyacrylamide gels (SDS-PAGE). After electrophoresis, the proteins were transferred to PVDF membrane filters (Millipore Biotechnology, Billerica, MA, USA). The membranes were incubated overnight at $4{ }^{\circ} \mathrm{C}$ with primary rabbit polyclonal VEGF-A antibodies (Immunoway Biotechnology Company, Newark, DE, USA) or primary rabbit polyclonal TIMP-1 antibodies (Immunoway Biotechnology Company, Newark, DE, USA). After washing three times in TBS-T, horseradish peroxidase (HRP)-conjugated secondary antibodies were used at a dilution of 1:5000 in TBS- $T$ for $2 \mathrm{~h}$ at room temperature. After three additional washes with TBS-T, the immunoreactive bands were visualised with a chemiluminescence reagent (ECL, Millipore Biotechnology, Billerica, MA, USA) and quanti- fied using a Bio-Rad imaging system (Bio-Rad Laboratories, Inc, Hertfordshire, UK).

\section{Statistical analysis}

SPSS software version 14.0 Sciences (SPSS Inc, USA) was used for statistical evaluation. Mann-Whitney U and Student's tests were used for comparing the groups. A probability value of $\mathrm{P}<0.05$ was considered significant.

\section{Results}

Formation of hypertrophic scars on rabbit ears

After 25 days, 128 HSs on the rabbit ears were successfully formed based on the rule of SEI $>1$. The HSs were erythematous, highly elevated from surrounding tissue, and stiff upon palpation (Figure 2). Afterwards, the HSs were divided into two groups and treated with either Endostar or saline.

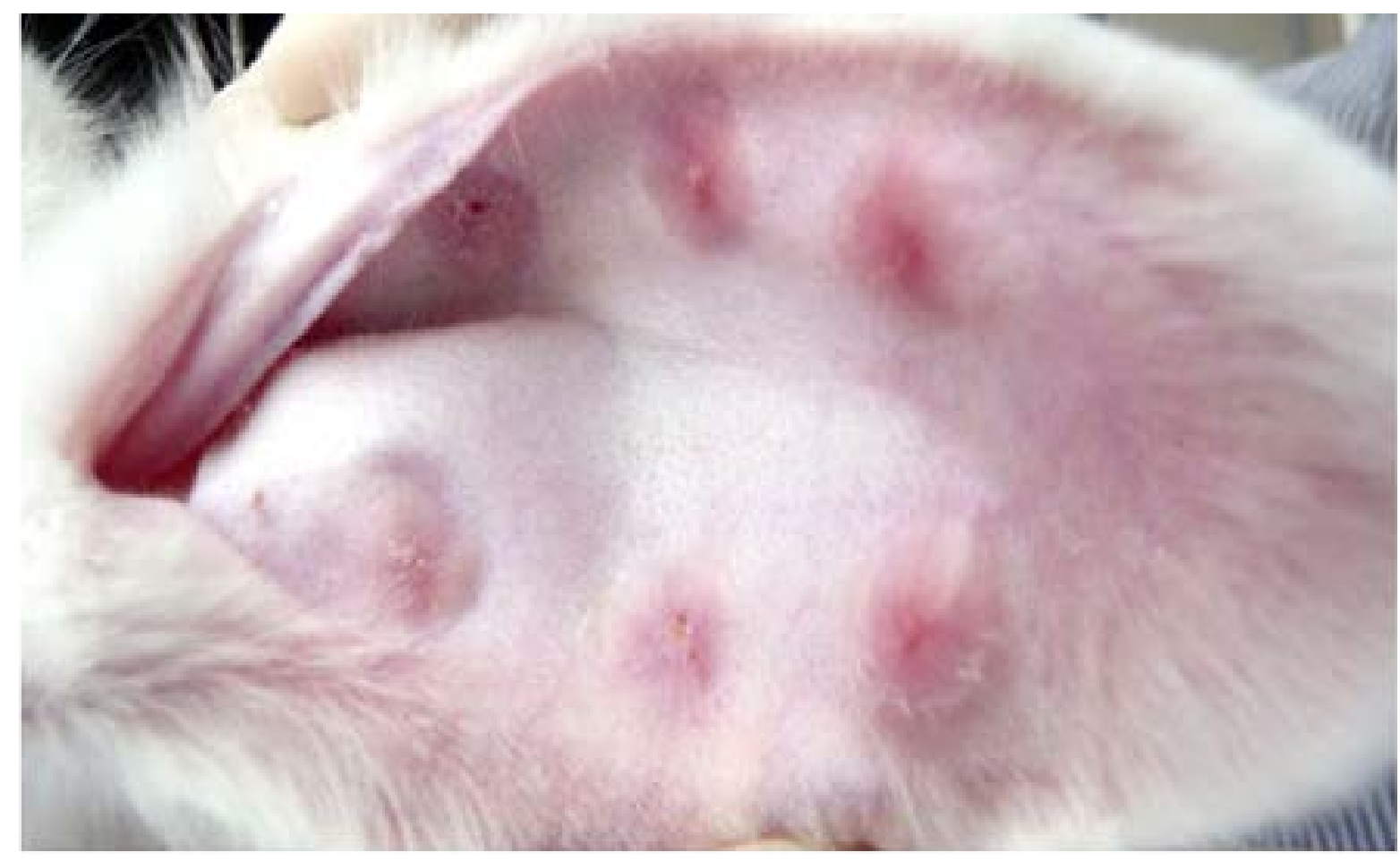

Figure 2. HS on rabbit ear successfully established after 25 days

Endostar improves appearance of scar

As shown in Figure 3A and Figure 3B, scars in the End- ostar group were softer, less erythematous, and thinner when compared to the control group after treatment with Endostar. 


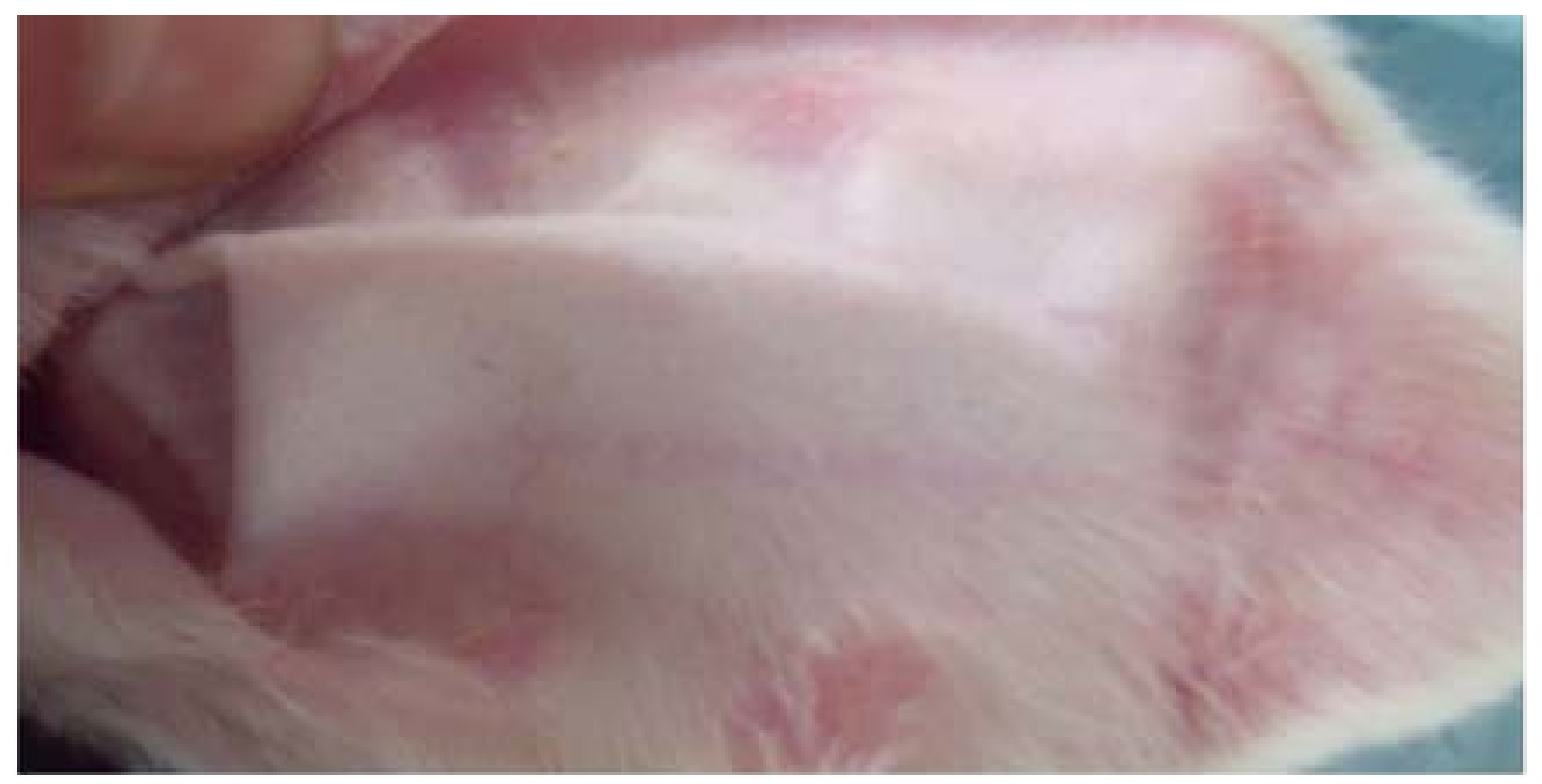

Figure 3. Endostar improved the appearance of HS: A) Appcarance of scar in the Endostar group:

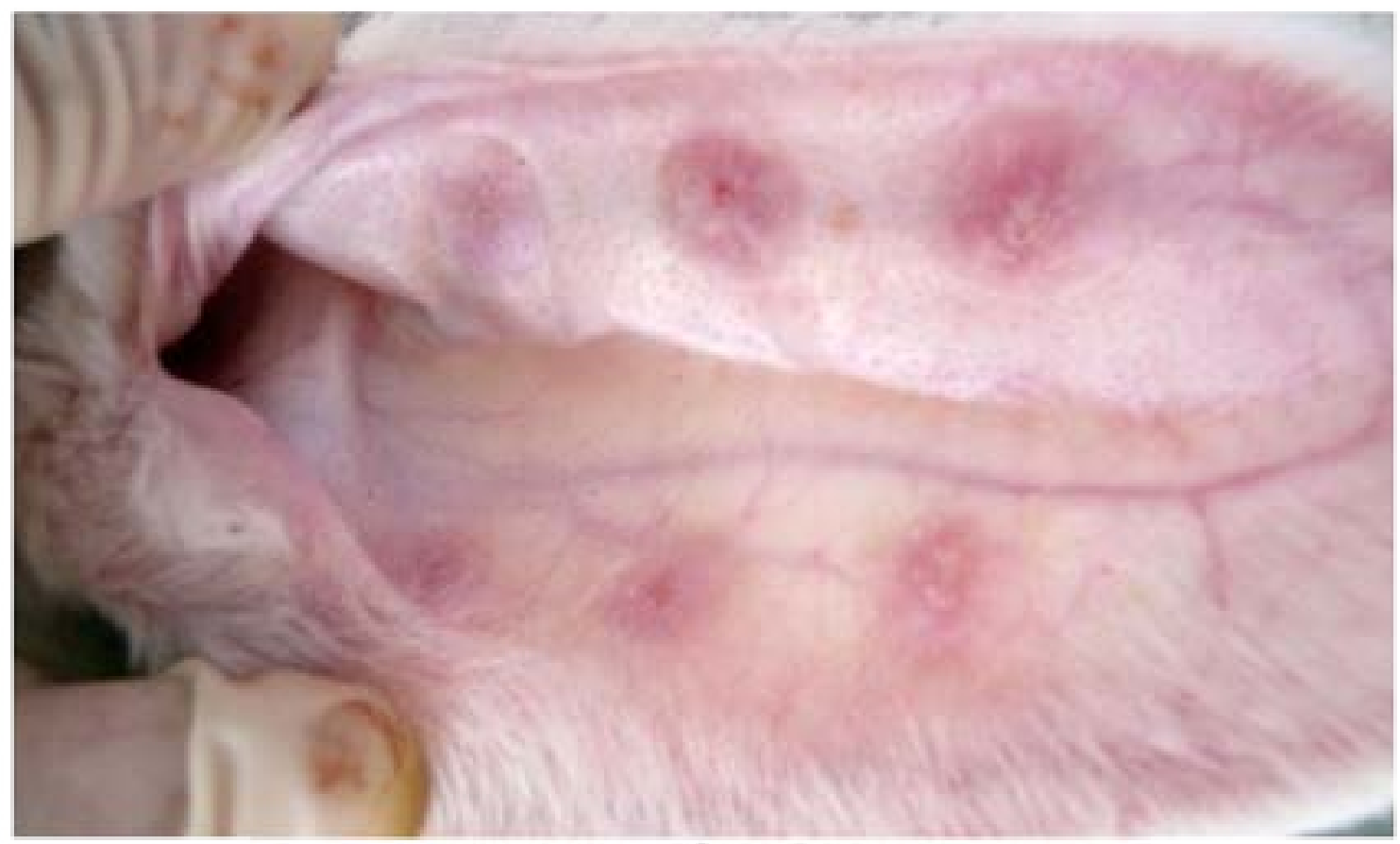

Figure 3B. Appearance of scar in the control group.

\section{Histology changes and scar index}

To investigate whether Endostar affected the histology of HS, HE staining was conducted. As shown in Figures $4 \mathrm{~A}$ and $4 \mathrm{~B}$, scars in the Endostar group had thinner connective tissue and less capillary and fibroblasts than those in the control group. Moreover, the distribution of col- lagen fibers in the Endostar group was regular, while they were littery and circinate in the control group. To better understand these changes, the scars were harvested for SEI measurement. It was discovered that the SEI of the Endostar group was significantly lower than that of the control group. SEI in the Endostar group was $1.37 \pm 0.21$ compared to $2.65 \pm 0.21$ in the control group $(\mathrm{P}<0.05)$. 


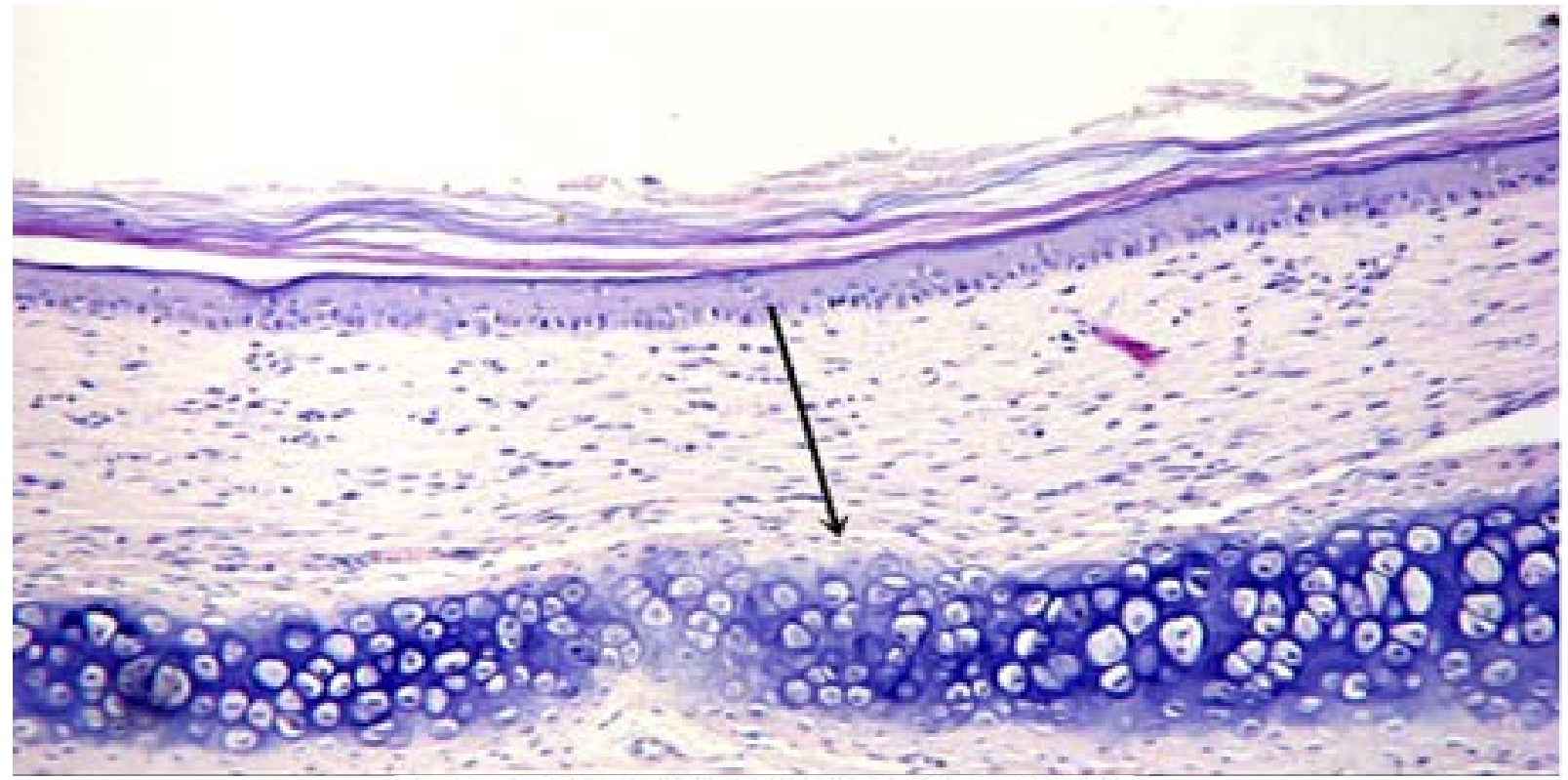

Figure 4A. Endostar group $(\mathrm{HE} \times 200)$.

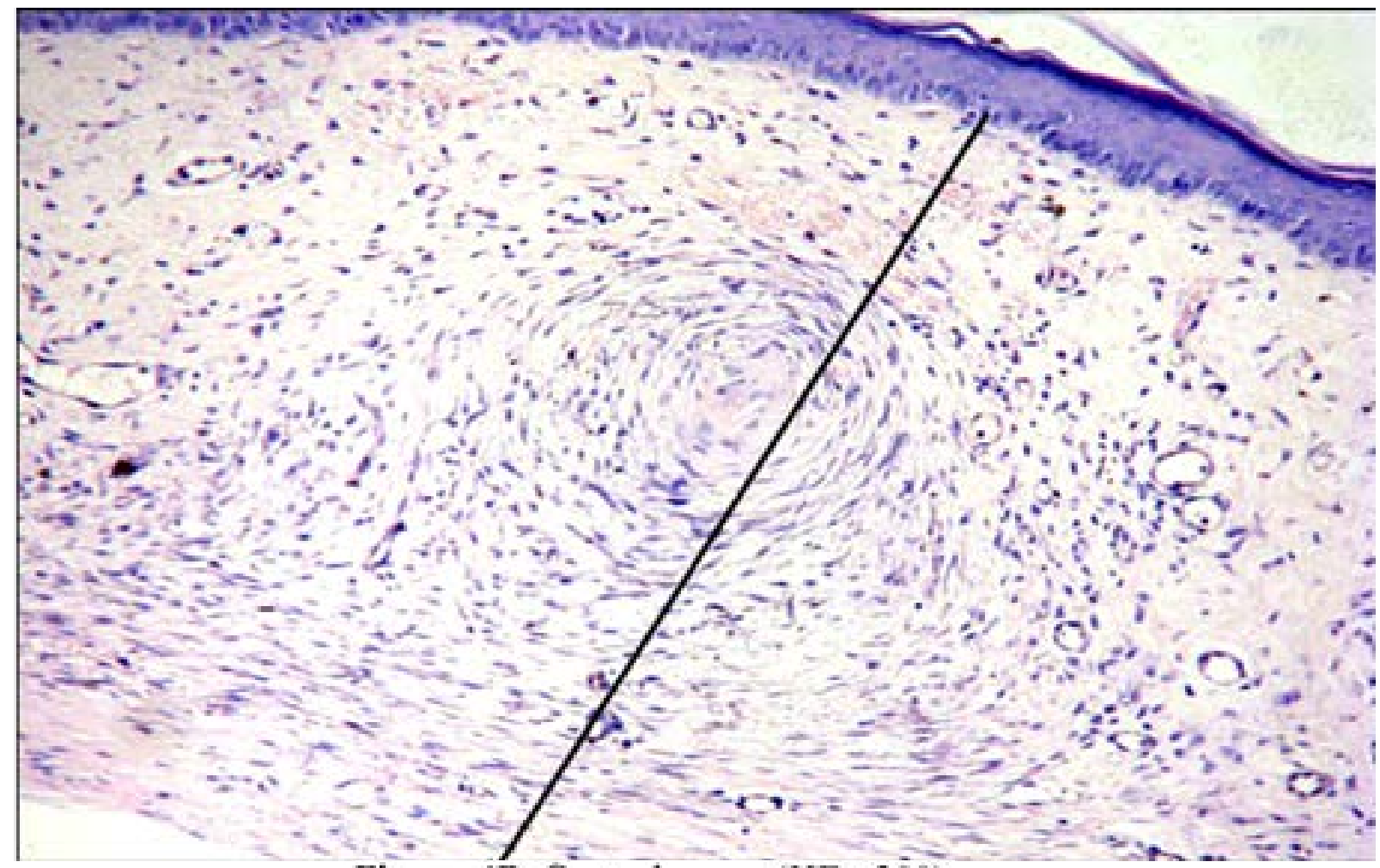

Figure 4B. Control group $(\mathrm{HE} \times 200)$.

\section{Endostar down-regulates mRNA expressions of VEGF and TIMP-1}

To determine the effects of Endostar on the VEGF and TIMP-1 mRNA expressions, RT-PCR was used to analyze their levels in the scar tissues. The relative expression of VEGF was $1.00 \pm 0.07$ and $0.78 \pm 0.16$ in the control group and Endostar group, respectively (Figure 5A). The relative expression of TIMP-1 was $1.00 \pm 0.05$ and 0.64 \pm 0.15 in the control group and Endostar group, respectively (Figure 5B). These results showed that Endostar significantly suppressed the expressions of VEGF and TIMP-1 mRNA. 


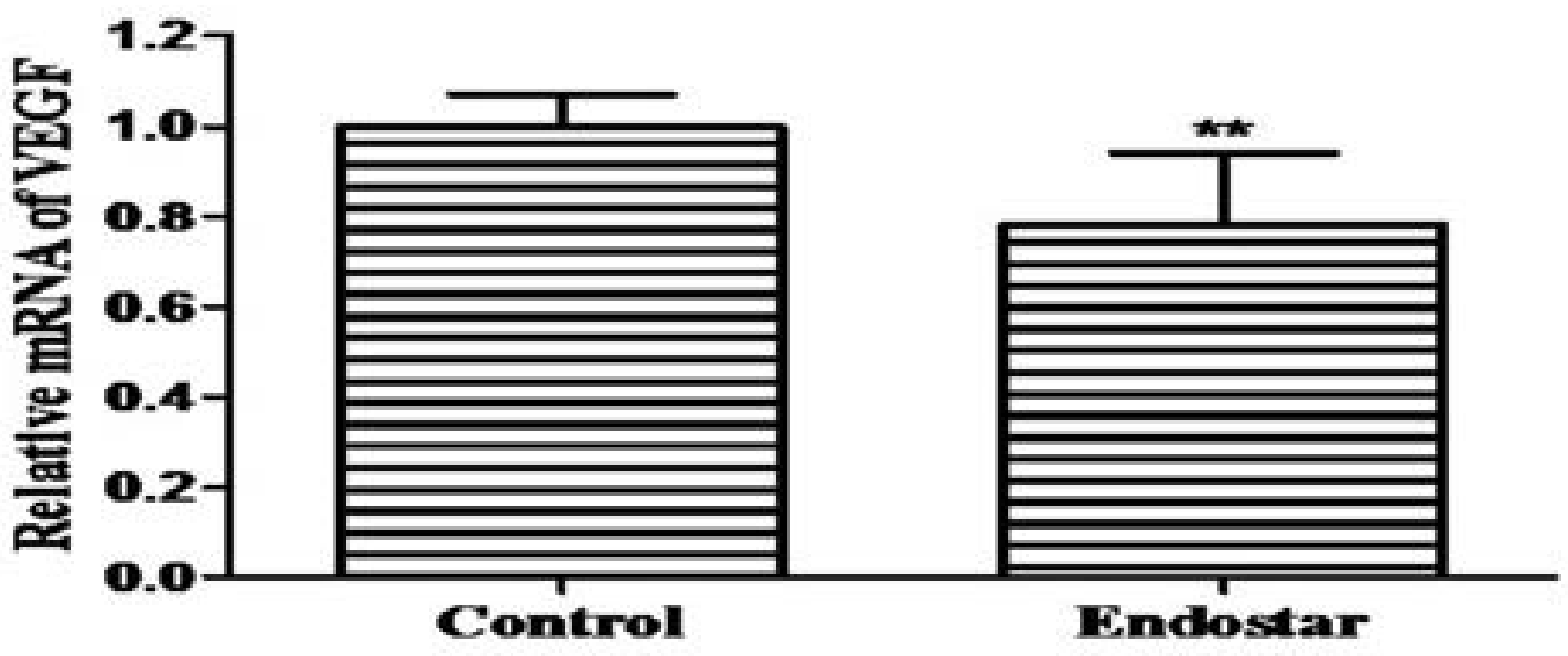

Figure 5A. mRNA expression of VEGF.

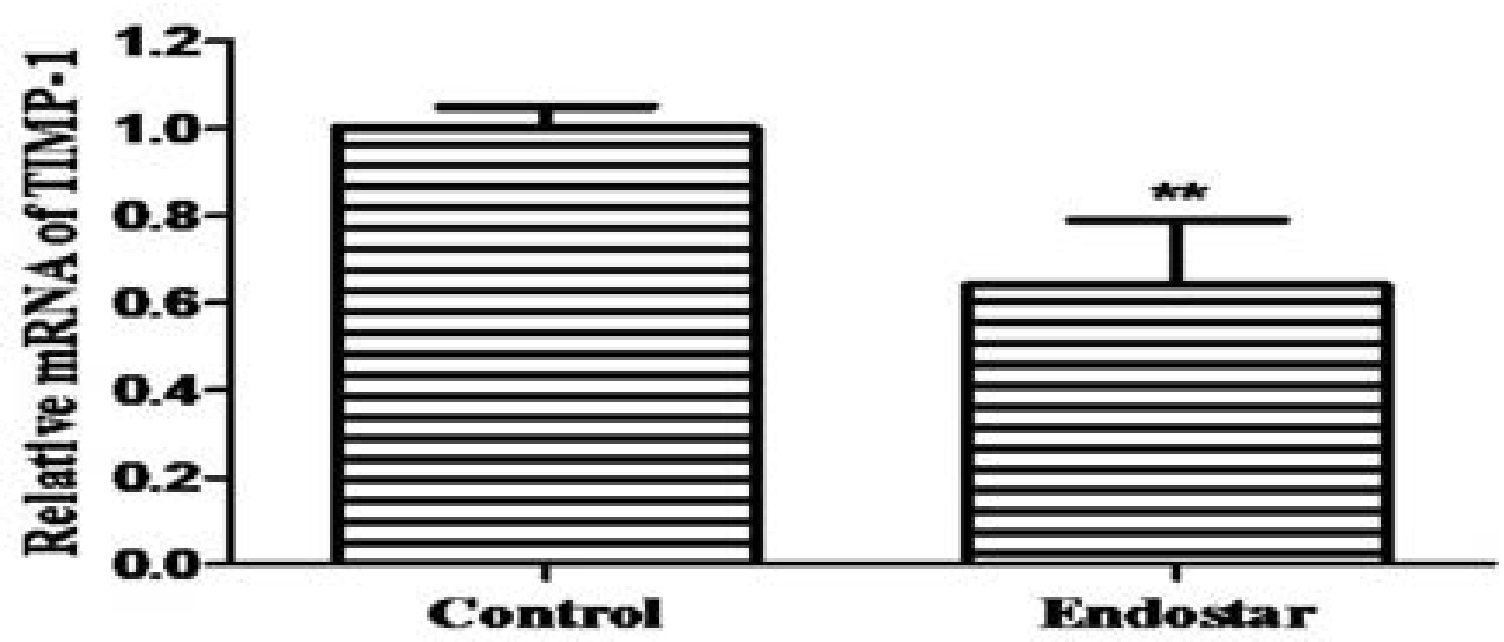

Figure 5B. mRNA expression of TIMP-1.

Endostar down-regulated protein expressions of VEGF and TIMP-1

Firstly, sixty-four scars in the Endostar group and sixtyfour scars in the control group were tested for VEGF and
TIMP-1 expressions by immunohistochemistry with their specific antibodies. Positive staining of VEGF was observed in $92.2 \%$ (59/64) scars in the control group, while there were only $59.4 \%$ (38/64) observed in the Endostargroup (Figures 6A, 6B). 


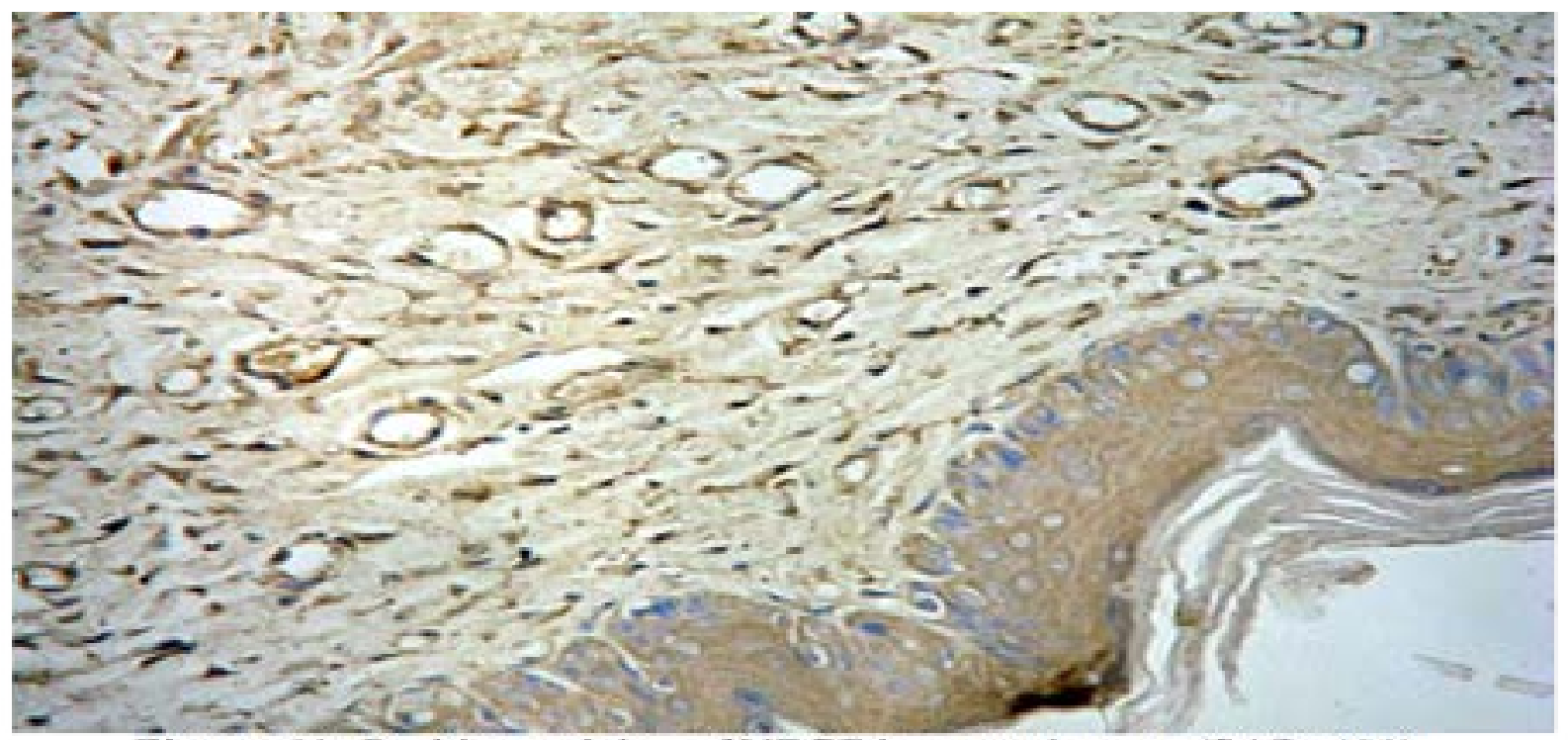

Figure 6A. Positive staining of VEGF in control group $(\mathrm{SAB} \times 400)$.

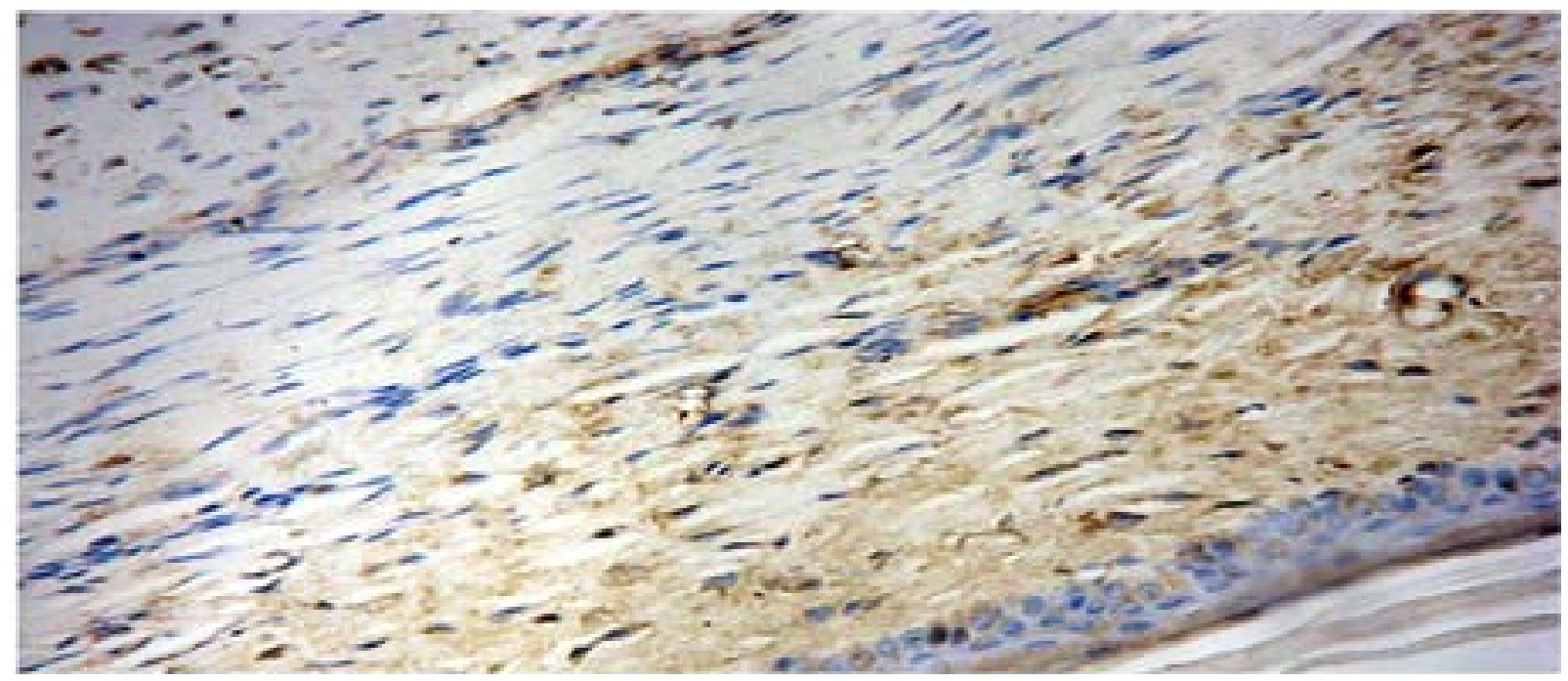

Figure 6B. Positive staining of VEGF in Endostar group (SAB $\times 400)$.

TIMP-1 was expressed in $87.5 \%(56 / 64)$ of the scars in the control group, and only $56.3 \%(36 / 64)$ of the scars in the Endostar group (Figure 6C,Figure 6D). 


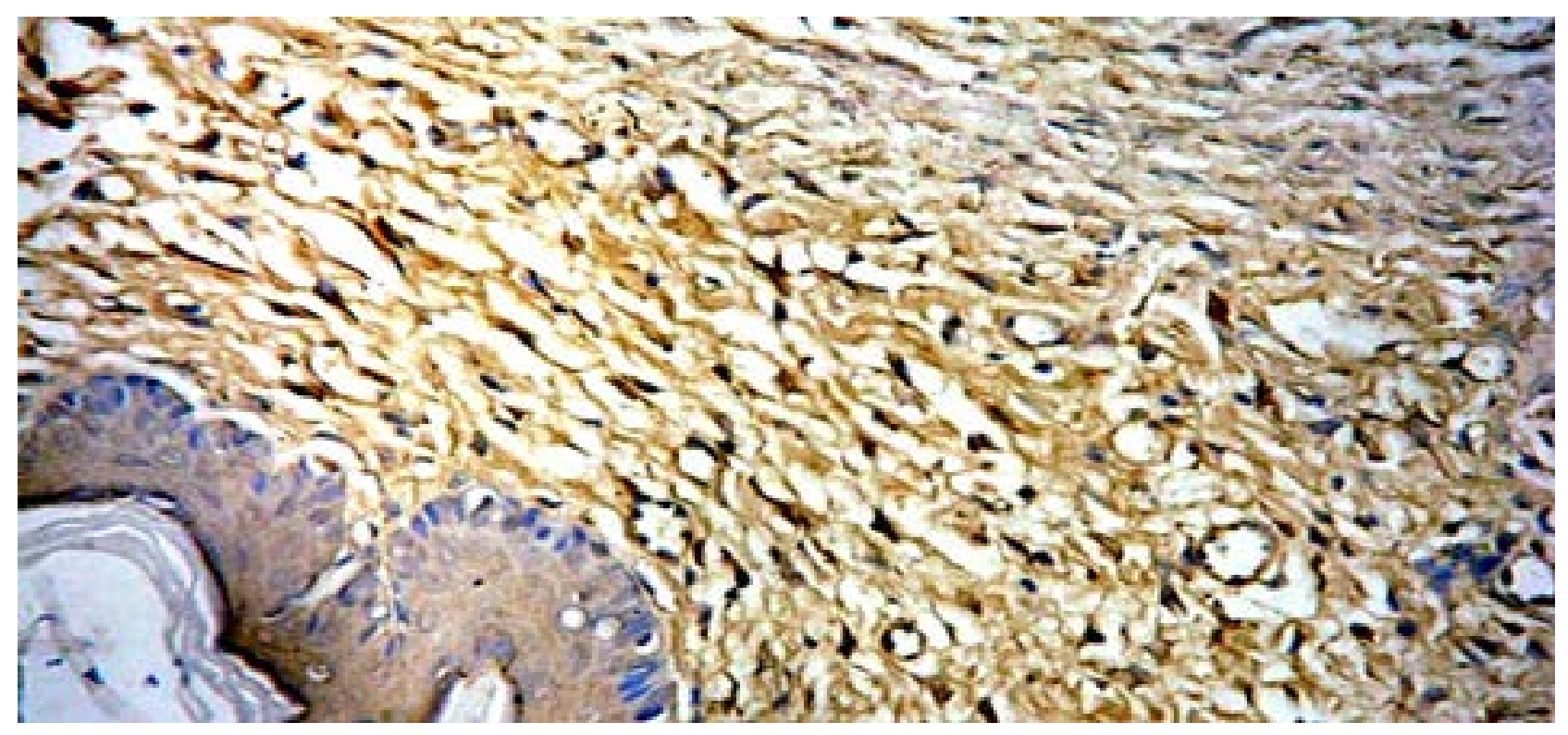

Figure 6C. Positive staining of TIMP-1 in control group $(\mathrm{SAB} \times 400)$.

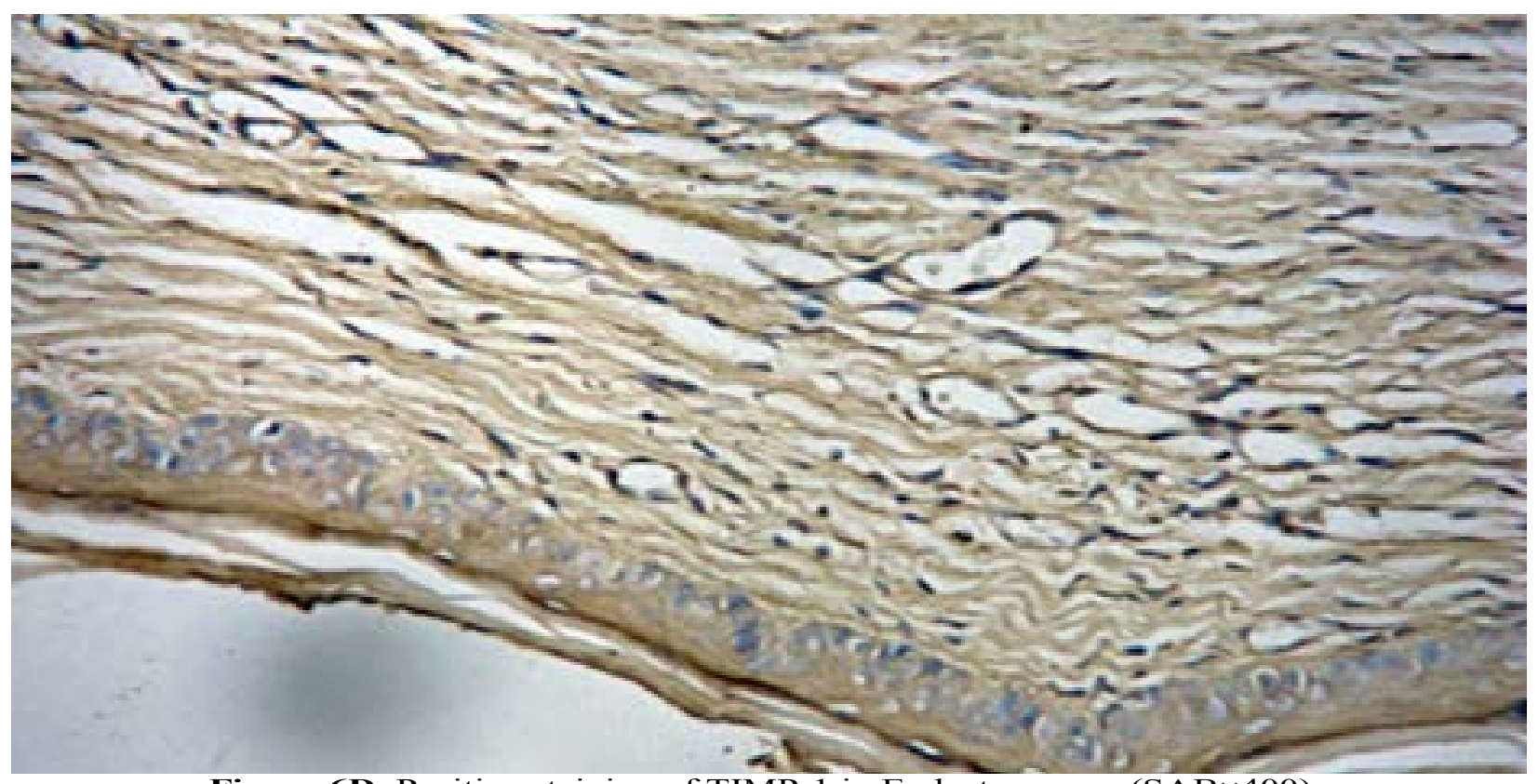

Figure 6D. Positive staining of TIMP-1 in Endostar group $(\mathrm{SAB} \times 400)$.

These results showed that the protein expressions of VEGF and TIMP-1 were significantly down-regulated by Endostar (both $\mathrm{P}<0.05)$ (Table 1).

Table I. Expression of VEGF and TIMP-1 in scar

\begin{tabular}{|c|c|c|c|c|c|c|c|c|c|}
\hline \multirow[b]{2}{*}{ Type } & \multirow[b]{2}{*}{$\mathrm{n}$} & \multicolumn{3}{|c|}{ VEGF } & \multirow[b]{2}{*}{$P$ value } & \multicolumn{3}{|c|}{ TIMP-1 } & \multirow[b]{2}{*}{$\mathrm{P}$ value } \\
\hline & & - & + & ++ & & - & + & ++ & \\
\hline Endostar & 64 & 26 & 33 & 5 & $<0.05$ & 28 & 31 & 5 & $<0.05$ \\
\hline Control & 64 & 5 & 46 & 13 & & 8 & 40 & 16 & \\
\hline
\end{tabular}


Thereafter, to verify the above results, protein expressions of VEGF and TIMP-1 were further detected by western blot. These results also suggested that Endostar significantly decreased the expressions of VEGF and TIMP-1, compared to the scars in the control group (Figure 7).

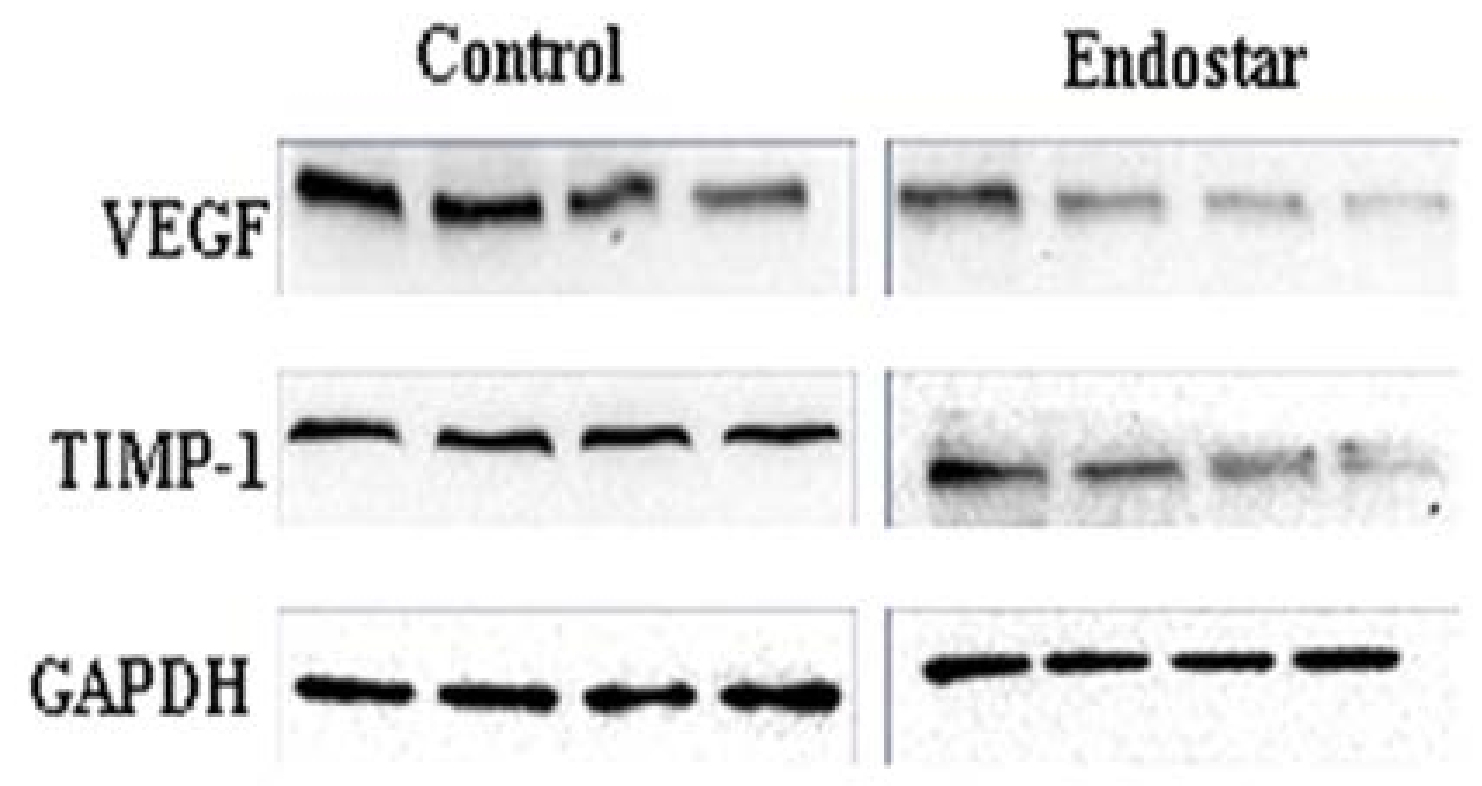

Figure 7. Expressions of VEGF and TIMP-1 Proteins in HS

\section{Discussion}

Altered appearance and function seriously affect the lives of HS patients. In addition, no single treatment appears to be effective for all HS sufferers. However, Endostar is a potent inhibitor of angiogenesis and has been successfully used in carcinoma therapy for its anti-angiogenesis properties ${ }^{7}$. It also plays an important role in anti-fibrosis treatment ${ }^{16}$. For example, Endostar significantly decreases the expression of Collagen-I, Collagen-III, and TGF- $\beta 1$ in HS of rabbit ears ${ }^{17-18}$. Endostar also decreases the expressions of $\alpha$-SMA and CTGF in other models ${ }^{19-20}$. Because HS is also associated with increased angiogenesis and $\mathrm{ECM}^{21-23}$, it was chosen as the object of this study, which showed that Endostar significantly reduced the formation of HS in rabbit ears by down-regulating VEGF and TIMP-1 expressions.

In this study, Endostar was administrated to the rabbits after the formation of the scars rather than before because it was believed that Endostar could decrease the microvessel density of the granulation tissue and delay wound healing ${ }^{24}$. After treatment with Endostar, the thickness of the connective tissues in HS was thinner, the number of micro vessels and fibroblasts were fewer, and the collagen fibers were smoother than those in the control group. This suggested that Endostar had a significantly inhibitive effect on HS formation. VEGF monoclonal antibody, another anti-angiogenesis protocol, has been reported to inhibit collagen production and excessive scar growth in $\mathrm{HS}^{11,25}$. In this study, it was found that mRNA and protein expression of VEGF was significantly decreased following Endostar treatment. Therefore, it was concluded that the inhibition of HS formation with Endostar may partly result from the reduction of angiogenesis by anti-VEGF mechanism.

It is important to maintain a balance between the synthesis and degradation of ECM in wound healing ${ }^{26}$. Excessive synthesis or lessened degradation of ECM will result in hypertrophic scars ${ }^{23}$. TIMPs and MMPs play a critical role in the formation, alteration, and degradation of matrix proteins ${ }^{27-29}$. TIMP-1 can combine with most MMP active sites, thus inhibiting enzyme activity ${ }^{30-31}$. However, some research has found that the expression of TIMP-1 is significantly higher in the scar tissue of patients with HS than in scar tissues of patients with normotrophic scars $^{32}$. Moreover, targeting TIMP-1 using small interfering RNA has significant therapeutic potential as an approach to treating keloids ${ }^{33}$. In this study, the expression of TIMP-1 was significantly decreased with Endostar 
treatments, while the group that did not receive Endostar therapy showed over expression of TIMP-1. Thus, it was thought that down-regulated TIMP-1 by Endostar also contributed to the inhibition of HS formation.

\section{Conclusion}

The findings suggested that Endostar reduced the formation of HS by down-regulation of VEGF and TIMP-1 expressions. However, whether Endostar directly targeted TIMP-1 or by another pathway requires further investigation. Therefore, further studies are needed to elucidate the more precise molecular mechanism of Endostar on the inhibition of HS.

\section{Conflict of interest}

The authors have no conflict of interest.

\section{References}

1. Berman B, Flores F. The treatment of hypertrophic scars and keloids. Eur J Dermatol 1998; 8(8):591 -595.

2. Leventhal D, Furr M, Reiter D. Treatment of keloids and hypertrophic scars: a meta-analysis and review of the literature. Arch Facial Plast Surg 2006; 8(6):362-368.

3. Kischer CW. The micro vessels in hypertrophic scars, keloids and related lesions: a review. J Submicrosc Cytol Pathol 1992; 24(2):281-296.

4. Amadeu T, Braune A, Mandarim-de-Lacerda C, Porto LC, Desmouliere A, Costa A. Vascularization pattern in hypertrophic scars and keloids: a stereological analysis. Pathol ResPract 2003; 199(7):469-473.

5. Thomas DW, Hopkinson I, Harding KG, Shepherd JP. The pathogenesis of hypertrophic/keloid scarring. Int J Oral Maxillofac Surg 1994; 23(4):232-236.

6. Ehrlich HP, Kelley SF. Hypertrophic scar: an interruption in the remodeling of repair-a laser Doppler blood flow study. Plast Reconstr Surg 1992(6); 90:993-998.

7. Eder JP Jr, Supko JG, Clark JW, Puchalski TA, Garcia-Carbonero R, Ryan DP, et al. Phase I clinical trial of recombinant human endostatin administered as a short intravenous infusion repeated daily. J Clin Oncol 2002; 20(18):3772-3784.

8. Steinbrech DS, Mehrara BJ, Chau D, Rowe NM, Chin G, Lee T, et al. Hypoxia upregulates VEGF production in keloid fibroblasts. Ann Plast Surg 1999; 42(5):514 -519; discussion 519-520.

9. Bock O, Schmid-Ott G, Malewski P, Mrowietz U.
Quality of life of patients with keloid and hypertrophic scarring. Arch Dermatol Res 2006(10); 297:433-438.

10. Ong CT, Khoo YT, Tan EK, Mukhopadhyay A, Do DV, Han HC, et al. Epithelial-mesenchymal interactions in keloid pathogenesis modulate vascular endothelial growth factor expression and secretion. J Pathol 2007;211(1):95-108.

11. Yue YG,Jiang CW,Li PY, Zhou S.Influence of the VEGF antibody targeted vascular therapy on the expression ofcollagen type I in hyperplastic. Zhonghua Shao Shang Za Zhi.2006;22(6):427-430.

12. Ulrich D,Noah EM,von Heimburg D, Pallua N. TIMP-1, MMP-2, MMP-9, and PIIINP as serum markers for skin fibrosis in patients following severe burn trauma. Plast Reconstr Surg 2003; 111(4):1423-1431.

13. Morris DE, Wu L, Zhao LL, Bolton L, Roth SI, Ladin DA, et al. Acute and chronic animal models for excessive dermal scarring: Quantitative studies. Plast Reconstr Surg 1997; 100(3):674-681.

14. Marcus JR, Tyrone JW, Bonomo S, Xia Y, Mustoe TA. Cellular mechanisms for diminished scarring with aging. Plast Reconstr Surg 2000; 105(5):1591-1599.

15. Taylor CR. Quantifiable internal reference standards for immunohistochemistry: the measurement of quantity by weight. Appl Immunohistochem Mol Morphol 2006; 14(3):253-259.

16. Yamaguchi Y1, Feghali-Bostwick CA.Role of endostatin in fibroproliferative disorders.-as a candidate for anti-fibrosis therapy. Nihon Rinsho Meneki Gakkai Kaishi 2013;36(6):452-458.

17. Yu Jian, Zhang Xiao-ming, Huang Xue-ying,et al. Study of the inhibitory effects of recombinant human endostatin on hypertrophic scars. Acta Universitatis Medicinalis Anbui 2014;49(7): 891-895.

18. Zhang Xiao-ming,Yu Jian,Huang Xue-ying,etal.Effects of recombinant human endostatin on the expression ofvascular endothelial growth factor,transforming growth factor- $\beta 1$ and basic fibroblast growth factor on hypertrophic scar in rabbit ears. Acta Anatomica Sinica 2015;46(1):101-105.

19. Chen J,Liu DG, Yang G,et al. Endostar,a novel human recombinant endostatin, attenuates liver fibrosis in CCl4induced mice. Exp Biol Med 2014;239(8):998-1006.

20. WU Zhan-qing,MA Qiang,GAO Yu-feng. Changes of VEGF,HIF-1 $\alpha, \mathrm{OPN}$ and CTGF levels in serum of HCC patients after recombinant human endostatin com- 
bined with transcatheter arterial chemoembolization. Chinese Journal of Biochemical Pharmacentics 2015;35(6):98-101.

21. Senger DR, Galli SJ, Dvorak AM, et al. Tumor cells secrete a vascular permeability factor that promotes accumulation of ascites fluid. Science 1983,219(4587):983-985. 22. Leung DW, Cachianes G, Kuang WJ, etal. Vascular endothelial growth factor is a secreted angiogenic mitogen. Science 1989,246(4935): 1306-1309.

23. Ulrich D, Ulrich F, Unglaub F, et al. Matrix metalloproteinases and tissue inhibitors of metalloproteinases in patients with different types of scars and keloids. J Plast Reconstr Aesthet Surg 2010,63(6): 1015-1021.

24. Wu Yuan-yuan. The role of protein kinase C-ain recombinant human endostatin inhibiting angiogenesis on wound healing of rabbit ear. Lanzhou:Lanzhou University, 2013.

25. Shen R, Li TZ, Qi SH, Liang HZ, Xu YB, Xie JL, et al. A research of endothelial cell-targeted therapy for cure of hypertrophic scar. Zhonghua Zheng Xing Wai Ke Za Zhi 2003;19(4): 254-257.

26. Ramadori G,Knittel T,Saile B.Fibrosis and altered matrix synthesis. Digestion 1998,59(4): 372-375.

27. Gabbiani G.The myofibroblast in wound healing and fibrocontractive diseases. J Pathol 2003;200:500-503.

28. Hetzel M, Bachem M, Anders D et al.Different effects of growth factors on proliferation and matrix pro- duction of normal and fibrotic human lung fibroblasts. Lung 2005;183(4):225-237.

29. Mirastschijski U,Schnabel R,Claes J et al.Matrix metalloproteinase inhibition delays wound healing and blocks the latent transforming growth factor-beta1-promoted myofibroblast formation and function. Wound Repair Regen 2010;18(2):223-234.

30. V. Egea, S. Zahler, N. Rieth, et al., "Tissue inhibitor of metalloproteinase-1 (TIMP-1) regulates mesenchymal stem cells through let-7f microRNA and Wnt/ $\beta$-catenin signaling," Proceedings of the National Academy of Sciences of the United States of America 2012;109(6):E309316.

31. Umenishi F,Umeda M,Miyazaki K.Efficient purification of TIMP-2 from culture medium conditioned by human hepatoma cell line,and its inhibitory effects on metalloproteinases and in vitro tumor invasion. J Biochem 1991;110(2):189-195.

32. Ulrich D, Ulrich F, Unglaub F, Piatkowski A, Pallua N. Matrix metalloproteinases and tissue inhibitors of metalloproteinases in patients with different types of scars and keloids. J Plast Reconstr Aesthet Surg 2010; 63(6):1015-1021. 33. Aoki M, Miyake K, Ogawa R, Dohi T, Akaishi S, Hyakusoku H, et al. siRNA Knockdown of Tissue Inhibitor of Metalloproteinase-1 in Keloid Fibroblasts Leads to Degradation of Collagen Type I. J Invest Dermatol 2014 ;134(3):818-826. 